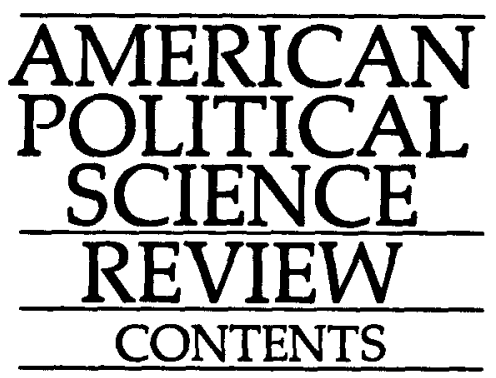

\title{
ARTICLES
}

Partisan Cycles in Congressional Elections and the Macroeconomy Alberto Alesina \& Howard Rosenthal

An Impression-driven Model of Candidate Evaluation Milton Lodge, Kathleen M. McGraw \& Patrick Stroh

A Schema-based Approach to Modeling an African-American Racial Belief System Richard L. Allen, Michael C. Dawson \& Ronald Brown

Life Span Civic Development and Voting Participation John Strate, Charles J. Parrish, Charles D. Elder \& Coit Ford III

Does Trade Still Follow the Flag?

Brian M. Pollins

Union Organization in Advanced Industrial Democracies Michael Wallerstein

Nuclear Deterrence and the Strategy of Limited Retaliation Robert Powell

The Political Economy of Military Spending in Israel Alex Mintz \& Michael D. Ward

Community, Integration, and Stability in Multinational Yugoslavia

Steven L. Burg \& Michael L. Berbaum

\section{RESEARCH NOTES}

Ideological Values and the Votes of U.S. Supreme Court Justices

Jeffrey A. Segal \& Albert D. Cover

Economic Conditions and the Presidential Vote

Robert S. Erikson

\section{CONTROVERSY}

Land Inequality and Political Violence Edward N. Muller, Mitchell A. Seligson, Hung-der Fu \& Manus I. Midlarsky 


\section{BOOK REVIEWS}

The Meaning of Democracy $\ldots \ldots \ldots \ldots \ldots \ldots \ldots \ldots \ldots \ldots \ldots \ldots \ldots \ldots, 603$

Political Theory $\ldots \ldots \ldots \ldots \ldots \ldots \ldots \ldots \ldots \ldots \ldots \ldots \ldots \ldots \ldots \ldots, 610$

American Politics $\ldots \ldots \ldots \ldots \ldots \ldots \ldots \ldots \ldots \ldots \ldots \ldots \ldots \ldots \ldots, 626$

Comparative Politics $\ldots \ldots \ldots \ldots \ldots \ldots \ldots \ldots \ldots \ldots \ldots \ldots \ldots \ldots \ldots, 655$

International Relations $\ldots \ldots, \ldots, \ldots \ldots \ldots \ldots \ldots \ldots \ldots \ldots \ldots \ldots \ldots, 680$

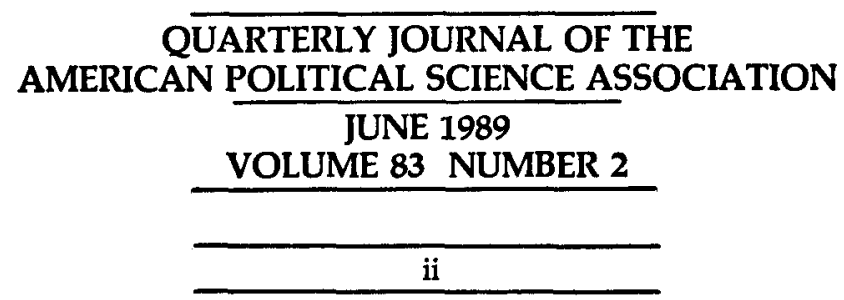




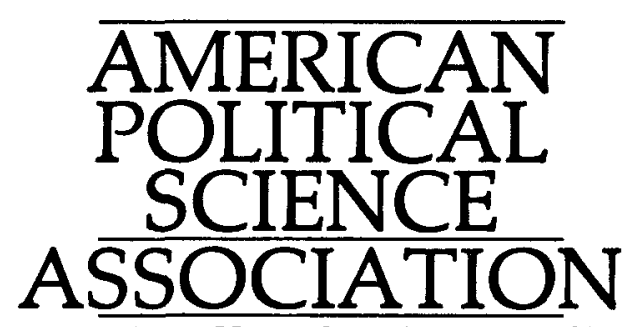

1527 New Hampshire Avenue, N.W. Washington, D.C. 20036

PRESIDENT:

Lucian W. Pye

Massachusetts Institute of Technology

PRESIDENT-ELECT:

Judith N. Shklar

Harvard University

VICE-PRESIDENTS:

Murray Edelman

University of Wisconsin

Robert Jervis

Columbia University

Martin Shapiro

University of California, Berkeley

SECRETARY:

Daniel J. Elazar

Temple University

TREASURER:

Nancy H. Zingale

College of St. Thomas

EXECUTIVE DIRECTOR:

Catherine E. Rudder

PROGRAM CHAIR:

Nelson W. Polsby

University of California, Berkeley

MANAGING EDITOR, APSR:

Samuel C. Patterson

Ohio State University
COUNCIL, 1987-89:

William I. Bacchus

Department of State

Donald Kinder

University of Michigan

Jane Mansbridge

Northwestern University

Dianne Pinderhughes

University of Illinois, Urbana-Champaign

Margaret E. Scranton

University of Arkansas, Little Rock

Mary Shanley

Vassar College

Richard A. Watson

University of Missouri, Columbia

Aristide Zolberg

New School for Social Research

COUNCIL, 1988-90:

Christopher Achen

University of Chicago

Kathleen Frankovic

CBS News, New York City

Peter A. Gourevitch

University of California, San Diego

Ted Robert Gurr

University of Colorado

Ronald Kahn

Oberlin College

Barbara J. Nelson

University of Minnesota

Adolph Reed, Jr.

Yale University

Donald Searing

University of North Carolina 


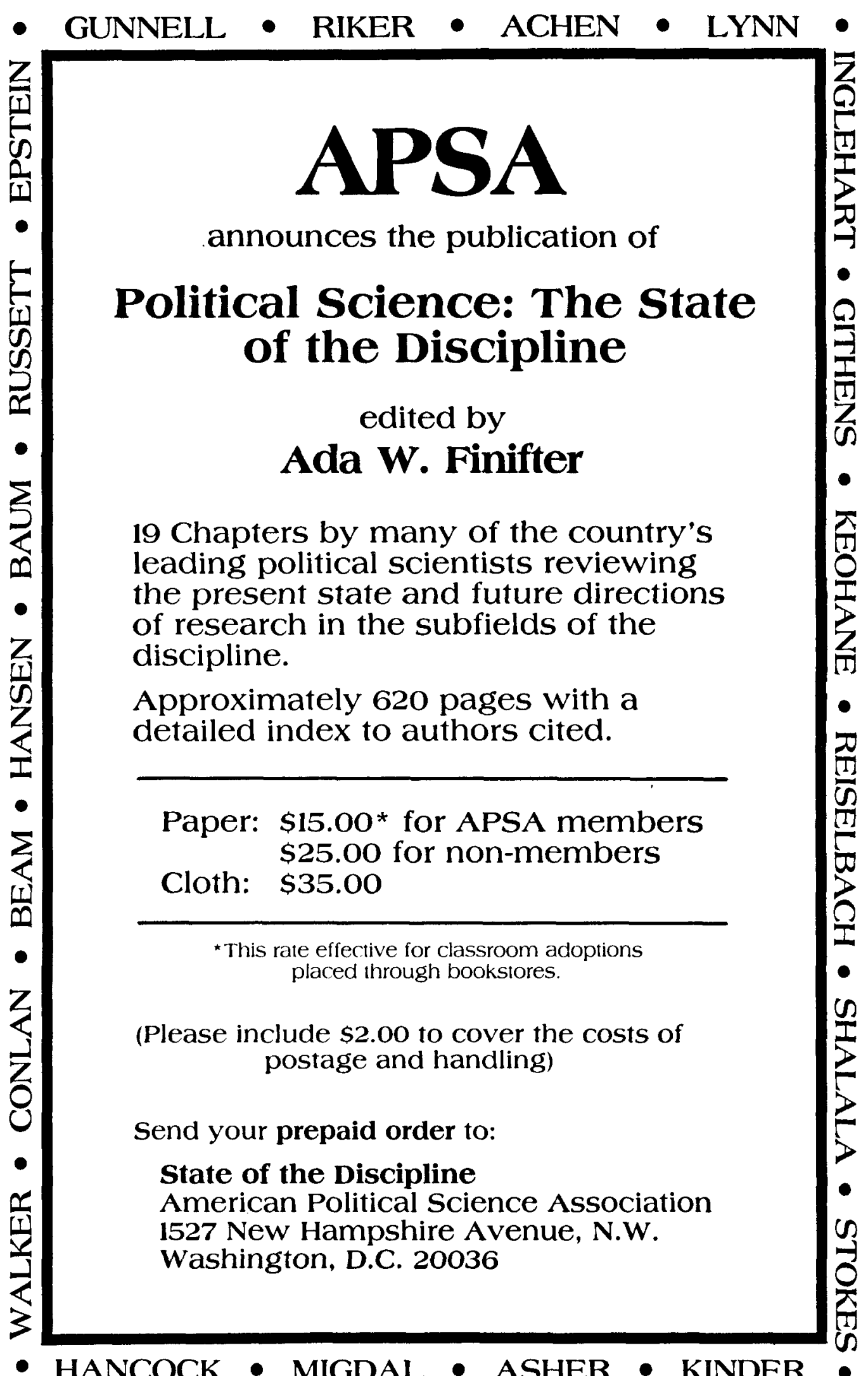

